\title{
PARTIAL DIFFERENTIAL EQUATIONS IN FISCHER-FOCK SPACES FOR THE HILBERT-SCHMIDT HOLOMORPHY TYPE
}

\author{
BY THOMAS A. W. DWYER, III ${ }^{1}$
}

Communicated by Philip Hartman, March 29, 1971

1. Introduction. Current work on the extension of function theory to infinite-dimensional domains has led to the consideration of classes of analytic functions defined on Banach spaces, with Fréchet derivatives of a given type, e.g., nuclear, compact or integral. The existence theory of partial differential equations in this setting follows from [G] for the nuclear bounded case, and is given in [D] for formal power series of $\alpha-\beta$ - $\gamma$-type. In this note we describe the duality theory (Theorem 1) and the existence theory (Theorem 2) of partial differential equations for a class of spaces of entire functions defined on a Hilbert space, with Fréchet derivatives given by Hilbert-Schmidt operators. When the underlying Hilbert space is finite-dimensional, we recover results in [T, Chapter 9], in [B] and in [NS] (Fischer space). When the underlying space is a Hilbert space of squareintegrable functions, we obtain the wave functionals in the Fock representation of quantum field theory (cf. [NT]), subsuming some of the results proved independently in [R].

2. Hilbert-Schmidt polynomials. Let $E$ be a Hilbert space over the complex field $C$, with inner product $(\mid)$, and $E^{\prime}$ the dual of $E$, with the inner product $\left(u^{\prime} \mid v^{\prime}\right)=(v \mid u)$ for $u^{\prime}=(\mid u), v^{\prime}=(\mid v)$. Let $E^{\prime v_{n}}$ $=E^{\prime} \bigvee \cdots \vee E^{\prime}$ denote the $n$-fold symmetric product of $E^{\prime}$ [Gr, p. 191]. The Hilbert-Schmidt inner product on $E^{\prime V_{n}}$ is characterized for decomposable elements by

$$
\left(u_{1}^{\prime} \vee \cdots \vee u_{n}^{\prime} \mid v_{1}^{\prime} \vee \cdots \vee v_{n}^{\prime}\right)=\frac{1}{n !} \sum_{\pi}\left(u_{\pi 1}^{\prime} \mid v_{1}^{\prime}\right) \cdots\left(u_{\pi n}^{\prime} \mid v_{n}^{\prime}\right),
$$

the summation extended over all permutations $\pi$ of the indices. $E_{H}^{\prime v_{n}}$ denotes the $n$-fold symmetric product equipped with the Hilbert-

AMS 1969 subject classifications. Primary 4630, 4645; Secondary 3205, 3597.

Key words and phrases. Entire functions, holomorphy type, Hilbert-Schmidt polynomials, partial differential operators, Fourier-Borel transform, Fock representation.

1 This work was derived from the author's doctoral dissertation, written under Professor John Horvath (University of Maryland, 1970). It was supported in part by an NSF Traineeship. 
Schmidt inner product, and $\left(E_{H}^{\prime \vee n}\right)^{\wedge}$ its completion.

For $n=1,2, \cdots$, let $\odot\left({ }^{n} E\right)$ denote the Banach space of continuous $n$-homogeneous polynomials $P$ (obtained from continuous symmetric $n$-linear forms $A: E \times \cdots \times E \rightarrow C$ by $P(x)=A(x, \cdots, x)$ ), with the supremum norm on the unit ball of $E$, and let $\rho\left({ }^{0} E\right)=C[N$, p. 7].

Proposition 1. The formula $i\left(u_{1}^{\prime} \bigvee \cdots \vee u_{n}^{\prime}\right)=u_{1}^{\prime} \cdots u_{n}^{\prime}$, where $u_{1}^{\prime} \cdots u_{n}^{\prime}(x)=u_{1}^{\prime}(x) \cdots u_{n}^{\prime}(x)$ for $x \in E$ (also $\left.u^{\prime n}=u^{\prime} \cdots u^{\prime}\right)$, defines a linear injection from $E_{H}^{\prime \vee n}$ into $\odot\left({ }^{n} E\right)$. The continuous linear extension $\tilde{\imath}$ of $i$ to $\left(E_{H}^{\prime \vee n}\right)^{\wedge}$ is still injective. The image of $\tilde{\imath}$, denoted by $\mathcal{P}_{H}\left({ }^{n} E\right)$, is the Hilbert space of $n$-homogeneous Hilbert-Schmidt polynomials on $E$, with the inner product inherited from $E_{H}^{\prime \vee n}$ denoted by $(\mid)_{H}$ and the associated norm by \|\|$_{H}$. Let $\odot_{H}\left({ }^{n} E\right)^{\prime}$ be equipped with the dual inner product. Given $P_{n} \in \mathcal{P}_{H}\left({ }^{n} E\right)$, the formula $P_{n}^{\prime}\left(x^{\prime}\right)$ $=\left(x^{\prime n} \mid P_{n}\right)_{H}$, where $x \in E$ and $x^{\prime n}=(\mid x)^{\prime}$, defines $P_{n}^{\prime} \in \mathcal{P}_{H}\left({ }^{n} E^{\prime}\right)$, and the map $\left(\mid P_{n}\right)_{H} \mapsto P_{n}^{\prime}$ is a Hilbert space isomorphism.

\section{Entire functions of Hilbert-Schmidt type.}

Proposition 2. Given $\rho>0$, if $f_{n} \in \odot_{H}\left({ }^{n} E\right)$ for each $n$ and $\sum_{n=0}^{\infty} \rho^{n}\left\|f_{n}\right\|_{H}^{2} / n !<\infty$ then $f=\sum_{n=0}^{\infty} f_{n} / n !$ is an entire function of bounded type, i.e., $f$ takes bounded sets into bounded sets. If $\hat{d}^{n} f(x)$ denotes the nth derivative polynomial of $f$ at $x$ then $\hat{d}^{n} f(0)=f_{n}$. The class of such functions, denoted by $\mathfrak{F}_{\rho}(E)$, is a Hilbert space, with the inner product $(\mid)_{\rho}$ given by

$$
(f \mid g)_{\rho}=\sum_{n=0}^{\infty} \rho^{n} \frac{1}{n !}\left(\hat{d}^{n} f(0) \mid \hat{d}^{n} g(0)\right)_{H}
$$

and the associated norm denoted by \|\|$_{\rho}$. Clearly \|\|$_{\rho} \leqq\|\|_{\sigma}$ when $\rho \leqq \sigma$. Hence $\mathcal{F}_{\infty}(E)=\bigcap_{0<p<\infty} \mathfrak{F}_{\rho}(E)$ with the projective limit topology is a countably Hilbert space, thus a reflexive Fréchet space, and $\mathcal{F}_{0}(E)$ $=\mathrm{U}_{0<\rho<\infty} F_{\rho}(E)$ with the locally convex inductive limit topology is a bornological $(D F)$-space.

THEOREM 1. Let $0 \leqq \rho \leqq \infty$, with $\rho^{-1}=0$ or $\infty$ when $\rho=\infty$ or 0 . If $f=\sum_{n=0}^{\infty} f_{n} / n ! \in \mathcal{F}_{\rho}(E)$ and $g^{\prime}=\sum_{n=0}^{\infty} g_{n}^{\prime} / n ! \in \mathcal{F}_{\rho}-1\left(E^{\prime}\right)$, and if $g_{n}^{\prime}$ $\in \mathcal{P}_{H}\left({ }^{n} E^{\prime}\right)$ corresponds to $\left(\mid g_{n}\right)_{H} \in \mathcal{P}_{H}\left({ }^{n} E\right)^{\prime}$, then the series

$$
\left\langle f, g^{\prime}\right\rangle=\sum_{n=0}^{\infty} \frac{1}{n !}\left(f_{n} \mid g_{n}\right)_{H}
$$

defines a bilinear form, placing $\mathfrak{F}_{\rho}(E)$ and $\mathfrak{F}_{\rho^{-1}}\left(E^{\prime}\right)$ in separating duality. The map $g^{\prime} \mapsto\left\langle, g^{\prime}\right\rangle$ is a Hilbert space isomorphism (resp. a topological vector space isomorphism) from $\mathfrak{F}_{\rho}(E)^{\prime}$ onto $\mathcal{F}_{\rho-1}\left(E^{\prime}\right)$ when 
$0<\rho<\infty$ (resp. $\rho=0$ or $\infty)$, and is the inverse of the Fourier-Borel transformation [D].

Sketch of PROOF. Let $T \in \mathcal{F}_{\rho}(E)^{\prime}$. Since $P_{H}\left({ }^{n} E\right)$ is continuously imbedded in each $\mathcal{F}_{\rho}(E)$, the restriction of $T$ to $\mathcal{P}_{H}\left({ }^{n} E\right)$ belongs to $\odot_{H}\left({ }^{n} E\right)^{\prime}$, corresponding to $T_{n}^{\prime} \in \mathcal{P}_{H}\left(E^{\prime}\right)$ given by $T_{n}^{\prime}\left(x^{\prime}\right)=T\left(x^{\prime n}\right)$. The formula $\hat{T}\left(x^{\prime}\right)=T\left(\exp \circ x^{\prime}\right)$ defines the Fourier-Borel transform $\hat{T}: E^{\prime} \rightarrow \boldsymbol{C}$ of $T$. We have in fact $\hat{T}=\sum_{n=0}^{\infty} T_{n}^{\prime} / n ! \in \mathscr{F}_{\rho-1}\left(E^{\prime}\right)$. Conversely, given $g^{\prime} \in \mathcal{F}_{\rho}-1\left(E^{\prime}\right)$ each $\hat{d}^{n} g^{\prime}(0) \in \mathcal{P}_{H}\left({ }^{n} E^{\prime}\right)$ corresponds to a unique $\left(\mid g_{n}\right)_{H} \in \mathcal{P}_{H}\left({ }^{n} E\right)^{\prime}$. The formula

$$
T_{g^{\prime}}(f)=\sum_{n=0}^{\infty} \frac{1}{n !}\left(d^{n} f(0) \mid g_{n}\right)_{H}
$$

for $f \in \mathcal{F}_{\rho}(E)$ defines $T_{g^{\prime}} \in \mathcal{F}_{\rho}(E)^{\prime}$, and we get $\hat{T}_{g^{\prime}}=g^{\prime}$. This establishes the isomorphism of vector spaces and the duality $\left\langle f, g^{\prime}\right\rangle=T_{\sigma^{\prime}}(f)$. Moreover, $\left\|g^{\prime}\right\|_{\rho^{-1}}=\left\|T_{g^{\prime}}\right\|$ (dual norm in $F_{\rho}(E)^{\prime}$ ) when $0<\rho<\infty$. The continuity of the mappings from $\mathfrak{F}_{\infty}(E)^{\prime}$ (resp. $\mathcal{F}_{0}(E)^{\prime}$ ) onto $\mathfrak{F}_{0}\left(E^{\prime}\right)$ (resp. $\mathcal{F}_{\infty}\left(E^{\prime}\right)$ ) follows from the isometry from $\mathfrak{F}_{\rho}(E)^{\prime}$ onto $\mathfrak{F}_{\rho^{-1}}\left(E^{\prime}\right)$ for $0<\rho<\infty$ by the properties of countably Hilbert spaces, bornological (DF)-spaces and their duals.

CoRollary 1. $\mathcal{F}_{0}(E)$ is reflexive and complete. $\mathcal{F}_{\infty}(E)$ and $\mathcal{F}_{0}(E)$ are Montel spaces, in fact nuclear, if and only if $E$ is finite-dimensional.

SkETCH OF PROOF. The reflexivity, hence completeness, of the (DF)space $\mathfrak{F}_{0}(E)$ follows from the duality. In the finite-dimensional case the nuclearity of $\mathcal{F}_{\infty}(E)$, hence of $\mathcal{F}_{0}(E)$, comes from the nuclearity of the injections $\mathcal{F}_{\sigma}(E) \rightarrow \mathcal{F}_{\rho}(E)$ for $\rho<\sigma . E^{\prime}$ is a closed barrelled subspace of $\mathcal{F}_{\infty}(E)$ and of $\mathcal{F}_{0}(E)$, so these spaces cannot be Montel or nuclear in the infinite-dimensional case.

4. Partial differential operators of Hilbert-Schmidt type. To define partial differential operators we need the following inequality.

Proposition 3. If $0 \leqq k \leqq n$ and $P_{n} \in \mathcal{P}_{H}\left({ }^{n} E\right)$ then $\hat{d}^{k} P_{n}(x) \in \mathcal{P}_{H}\left({ }^{k} E\right)$, and for all $x \in E$ we have:

$$
\left\|\frac{1}{k !} \hat{d}^{k} P_{n}(x)\right\|_{H} \leqq\left(\begin{array}{l}
n \\
k
\end{array}\right)\left\|P_{n}\right\|_{H}\|x\|^{n-k} .
$$

The proof, and others below, makes use of the following representation:

LEMMA 1. Given an orthonormal basis $\left(e_{i}\right)_{i}$ of $E$, each $P_{n} \in \odot_{H}\left({ }^{n} E\right)$ is uniquely expressed as a limit in \|\|$_{H-n o r m}$ by 


$$
P_{n}=\sum_{i_{1}, \cdots, i_{n}} s_{i_{1}} \cdots i_{i_{n}} e_{i_{1}}^{\prime} \cdots \dot{e}_{i_{n}}^{\prime}
$$

with symmetric coefficients $s_{i_{1}} \cdots i_{i_{n}} \in C$, and

$$
\left\|P_{n}\right\|_{H}^{2}=\sum_{i_{1}, \cdots, i_{n}}\left|s_{i_{1}} \cdots i_{n}\right|^{2}
$$

We observe, however, that the $e_{i_{1}}^{\prime} \cdots e_{i_{n}}^{\prime}$ are not orthonormal.

By $\left[\mathrm{N}, \S 9\right.$, Lemma 1] we get $\left.\dot{d}^{n} f(x) \in \mathcal{P}_{H}{ }^{n} E\right)$ for all $x \in E$ when $d^{n} f(0) \in \mathcal{P}_{H}\left({ }^{n} E\right)$ and $\lim \sup _{n}\left\|\hat{d}^{n} f(0) / n !\right\|_{H}^{1 / n}=0$. We may then define: given $P=\sum_{n=0}^{m} P_{n}$ with $P_{n} \in \mathcal{P}_{H}\left({ }^{n} E\right)$, the partial differential operator of Hilbert-Schmidt type $P(d)$ is given by

$$
P(d) f(x)=\sum_{n=0}^{m}\left(\hat{d}^{n} f(x) \mid P_{n}\right)_{H} .
$$

If $P=u_{1}^{\prime} \cdots u_{n}^{\prime}$ then $P(d)$ is given by successive directional differentiation along $u_{1}, \cdots, u_{n}$. In particular, we are reduced to linear partial differential operators with constant coefficients in the finitedimensional case. We also define the multiplication operator $P$. by $P \cdot f(x)=P(x) f(x)$.

Proposition 4. If $f$ is in $\mathcal{F}_{\sigma}(E)$ then $P(d) f$ and $P \cdot f$ are in $\mathcal{F}_{\rho}(E)$ for every $0<\rho<\sigma<\infty$. Hence if $f$ is in $\mathcal{F}_{\infty}(E)\left(\right.$ resp. $\mathfrak{F}_{0}(E)$ ) then so are $P(d) f$ and $P \cdot f$.

Easy counterexamples show that not all $f \in \mathcal{F}_{\rho}(E)$ are mapped into $\mathcal{F}_{\rho}(E)$ by $P(d)$ or $P$. .

ThEOREM 2. Let $0 \leqq \rho \leqq \infty$ and let $P(d)$ be any partial differential operator of Hilbert-Schmidt type: then for every $f \in \mathcal{F}_{\rho}(E)$ there is some $g \in \mathcal{F}_{\rho}(E)$ such that $P(d) g=f$.

The proof uses the following lemmas:

Lemma 2. If $P=\sum_{n=0}^{m} P_{n}$ and $P^{\prime}=\sum_{n=0}^{m} P_{n}^{\prime}$, where $\left(\mid P_{n}\right)_{H}$ $\in \mathcal{P}_{H}\left({ }^{n} E\right)^{\prime}$ corresponds to $P_{n}^{\prime} \in \mathcal{P}_{H}\left({ }^{n} E^{\prime}\right)$ by $P_{n}^{\prime}\left(x^{\prime}\right)=\left(x^{\prime n} \mid P_{n}\right)_{H}$, then $\left\langle P(d) f, g^{\prime}\right\rangle=\left\langle f, P^{\prime} \cdot g^{\prime}\right\rangle$ for $f$ and $g^{\prime}$ in the corresponding dual pairs (Theorem 1), finiteness and equality holding when either side is finite.

The proof follows from a similar identity for the duality between $\mathcal{P}_{H}\left({ }^{n} E\right)$ and $\mathcal{P}_{H}\left({ }^{n} E^{\prime}\right)$, established first for polynomials of finite type (i.e., given by $E^{\prime \vee n}$ ), which are dense in $\rho_{H}\left({ }^{n} E\right)$.

Lemma 3. If $0<\rho<\infty, f \in \mathcal{F}_{\rho}(E)$ and $P=\sum_{n=0}^{m} P_{n}$ with $P_{n} \in \mathcal{P}_{H}\left({ }^{n} E\right)$ then $\|P \cdot f\|_{\rho} \geqq\left\|P_{m}\right\|_{\rho}\|f\|_{\rho}$. 
The proof of the inequality uses a polynomial identity given in [T, Lemma 2.2] applied first to $P$ of finite type and $f \in U_{n} \odot_{H}\left({ }^{n} E\right)$, extended by density to any Hilbert-Schmidt polynomial $P$, and finally to any $f \in \mathcal{F}_{\rho}(E)$, with the help of the following facts: the pairs of operators $e^{\prime}(d)$ and $e^{\prime}$. obtained from an orthonormal basis $\left(e_{i}^{\prime}\right)_{i}$ of $E^{\prime}$ satisfy the correct commutation relations required over the polynomials; and Taylor series converge in \|\|$_{\rho}$-norm. The continuity of $P \cdot f \mapsto f$ follows from the inequality, and from the properties of projective and inductive limits in the cases $\rho=\infty$ and $\rho=0$.

Proof of Theorem 2. By Lemma 2 the transpose of $P(d)$ by $\langle$,$\rangle is P^{\prime}$, which has a continuous left inverse by Lemma 3 applied to $\mathfrak{F}_{\rho^{-1}}\left(E^{\prime}\right)$ (again $\rho^{-1}=0$ when $\rho=\infty$ ). A standard HahnBanach argument gives the result.

Proposition 5. Let $M$ be a measure space (e.g., locally compact), and make $E=L^{2}(M)$ : then $P_{n} \in \mathcal{P}_{H}\left({ }^{n} E\right)$ if and only if there is some $h_{n} \in L^{2}(M \times \cdots \times M),(n$ variables and product measure $)$, such that

$$
P_{n}(\alpha)=\int_{M} \cdots \int_{M} h_{n}\left(t_{1}, \cdots, t_{n}\right) \alpha\left(t_{1}\right) \cdots \alpha\left(t_{n}\right) d t_{1} \cdots d t_{n}
$$

for every $\alpha \in L^{2}(M)$. The function $h_{n}$ can be uniquely chosen to be symmetric, and then $\left\|P_{n}\right\|_{H}=\left\|h_{n}\right\|_{L^{2}}$.

It follows that the functions $f \in \mathcal{F}_{\rho}(E)$ are the Fock functionals of [NT] and [R], and the partial differentials $P_{n}(d) f(\alpha)$ are the functional derivatives $h_{n} f^{(n)}(\alpha)$ of [R], where $h_{n}$ corresponds to $P_{n}$ by the formula given above. The proof of Proposition 5 follows from the Hilbert space isomorphism between $\left(L^{2}(M)_{H}^{\vee n}\right)^{\wedge}$ and symmetric $L^{2}(M \times \cdots \times M)$.

\section{REFERENCES}

[B] V. Bargmann, On a Hilbert space of analytic functions and an associated integral transform, Comm. Pure Appl. Math. 14 (1961), 187-214. MR 28 \#486.

$\longrightarrow$ - On a Hilbert space of analytic functions and an associated integral transform. II. A family of related function spaces. Application to distribution theory, Comm. Pure Appl. Math. 20 (1967), 1-101. MR 34 \#1836.

[D] S. Dineen, Holomorphic functions on a Banach space, Bull. Amer. Math. Soc. 76 (1970), 883-886.

[G] C. Gupta, On the Malgrange theorem for nuclearly entire functions of bounded type on a Banach space, Nederl. Akad. Wetensch. Proc. Ser. A 73 (1970), 356-358.

[Gr] W. H. Greub, Multilinear algebra, Die Grundlehren der math. Wissenschaften, Band 136, Springer-Verlag, New York, 1967. MR 37 \#222.

$[\mathrm{N}]$ L. Nachbin, Topology on spaces of holomorphic mappings, Ergebnisse der Mathematik und ihrer Grenzgebiete, Band 47, Springer-Verlag, New York, 1969. MR $40 \# 7787$. 
[NS] D. Newman and H. Shapiro, Certain Hilbert spaces of entire functions, Bull. Amer. Math. Soc. 72 (1966), 971-977. MR $34 \# 4890$.

[NT] Yu. V. Novozhilov and A. V. Tulub, The method of functionals in the quantum theory of fields, Uspehi Fiz. Nauk 61 (1957), 53-102; English transl., Russian Tracts on Advanced Math. and Phys., vol. 5, Gordon and Breach, New York, 1961. MR 25 \#959.

[R] J. Rzewuski, On Hilbert space of entire functionals, Bull. Acad. Polon. Sci. Sér. Sci. Math. Astronom. Phys. 17 (1969), 453-458; 459-466, 571-578. MR 40 \#6243; 6244; 6245.

[T] F. Trèves, Linear partial differential equations with constant coefficients: Existence, approximation and regularity of solutions, Math. and its Applications, vol. 6, Gordon and Breach, New York, 1966. MR 37 \#557.

Northern Illinois University, Dekalb, Illinois 60115 